\title{
Effect of Wavelength on Metrological Characteristics of Non-Holographic Fiber Specklegram Sensor
}

\author{
Victor H. ARISTIZABAL ${ }^{1 *}$, Alejandro HOYOS ${ }^{2}$, Edgar RUEDA $^{3}$, \\ Nelson D. GOMEZ ${ }^{4}$, and Jorge A. GOMEZ ${ }^{2}$ \\ ${ }^{1}$ Grupo de Sistemas en Operaciones y Desarrollo Aplicado -SODA-, Universidad Cooperativa de Colombia, Cll 50A \\ \#41-40, Medellín, Colombia \\ ${ }^{2}$ Grupo de Física Básica y Aplicada, Politécnico Colombiano Jaime Isaza Cadavid, Cra 48 \#7-151, Medellín, Colombia \\ ${ }^{3}$ Grupo de Óptica y Fotónica, Instituto de Física, Universidad de Antioquia, Cll 70No. 52-21, Medellín, Colombia \\ ${ }^{4}$ Grupo de Investigación en Automática, Electrónica y Ciencias Computacionales-AE\&CC-, Instituto Tecnológico \\ Metropolitano - Institución Universitaria, Cll 54A \#30-01, Medellín, Colombia \\ *Corresponding author: Victor H. ARISTIZABAL_Ｅ-mail: vharisti@yahoo.com
}

\begin{abstract}
In this paper, we report some results about the effects of varying the wavelength in a structure of a non-holographic fiber specklegram sensor. In these arrangements, the speckle pattern produced by a multi-mode optical fiber is coupled to the asingle-mode optical fiber with lower numerical aperture, which produces a filtering effect that can be used as an optical transduction mechanism. The influence of the wavelength on the sensor performance is evaluated by changing the laser wavelength, and a strong effect on the linearity and reproducibility of its response is found. Lasers emitting at $1310 \mathrm{~nm}, 1550 \mathrm{~nm}$, and $1625 \mathrm{~nm}$ are used.
\end{abstract}

Keywords: Optical sensing, speckle sensor, optical fiber sensor, mechanical perturbation sensor

Citation: Victor H. ARISTIZABAL, Alejandro HOYOS, Edgar RUEDA, Nelson D. GOMEZ, and Jorge A. GOMEZ, "Effect of Wavelength on Metrological Characteristics of Non-Holographic Fiber Specklegram Sensor," Photonic Sensors, 2015, 5(1): $1-5$.

\section{Introduction}

When laser radiation is launched in a multimode optical fiber, at the output end of the fiber a complex speckle pattern appears, which is known as modal noise in optical communication systems, and it is an undesired effect on data transmission [1]. Fiber speckle patterns contain information of the spatial state of the optical fiber, which can be used in metrological applications. Small perturbations on the multimode optical fiber will produce changes in the spatial distribution of the speckle pattern which can be detected by optical correlation techniques or by intensity changes [2, 3]. Sensing systems based on fiber-speckle are known as the fiber specklegram sensor (FSS).

Recently, we explored an FSS based on optical power detection for monitoring high frequency mechanical disturbances and demonstrated that the numerical aperture of the multimode optical fiber has a strong effect on the reproducibility of the mechanical perturbations, i.e., the near field speckle size has a strong effect on the metrological characteristics of the non-holographic FSS. The

Received: 4 July 2014 / Revised version: 8 December 2014

(C) The Author(s) 2014. This article is published with open access at Springerlink.com

DOI: 10.1007/s13320-014-0210-3

Article type: Regular 
mechanism to explain the metrological effects, is based on an increase in the average speckle size of the speckle pattern generated at the output of a multimode fiber, which increases when the numerical aperture of the multimode fiber decreases [4]. As the speckle size generated in the multimode fiber depends directly on the wavelength, in this work, we explored the effects of changing the operation wavelength of the sensor, which consequentially, modified the geometric characteristics of the fiber speckle pattern. An increase in the sensor linearity response was observed for higher wavelengths.

\section{Theoretical analysis}

When laser radiation of the wavelength $\lambda$ is inserted into a multimode optical fiber, at the output of the fiber, the spatial intensity distribution is the superposition of optical modes traveling through the fiber. In the case of a step profile optical fiber, the number of modes $M$ is obtained from the optical fiber normalized frequency: $V=k_{0} a \sqrt{n_{\mathrm{co}}^{2}-n_{\mathrm{cla}}^{2}} \approx$ $k_{0} a(A N)$, where $n_{\text {co }}$ and $n_{\text {cla }}$ are the core and cladding refractive indices, respectively; $a$ is the radius, and $A N=\sqrt{n_{\mathrm{co}}^{2}-n_{\mathrm{cla}}^{2}}$ is the numerical aperture of the optical fiber; $k_{0}=2 \pi / \lambda i s$ is the wavenumber of light in vacuum.

When the radius of the fiber is much greater than the wavelength of laser radiation, i.e., $a / \lambda \gg>1, M$ can be expressed as [5]

$$
M=V^{2} / 2 \text {. }
$$

In near field approximation, the fiber speckle pattern has an average grain diameter given by [5]

$$
D=2 \pi^{2} a^{2} / M
$$

or in terms of the numerical aperture and wavelength:

$$
D=\lambda / A N \text {. }
$$

Equation (3) indicates the possibility to modify the geometrical characteristics of the speckle pattern by making changes in the numerical aperture of the multimode fiber or by changing the wavelength of the laser radiation. In the next section, we will evaluate experimentally the effects of the wavelength, which is equivalent to evaluating the average grain speckle diameter, on the performance of a non-holographic fiber specklegram sensor.

To simulate the speckle patterns, the propagation modes in the multimode fiber are calculated using the equation:

$$
\nabla \times \nabla \times \mathbf{E}-k_{0}^{2} n^{2} \mathbf{E}=0
$$

where $n$ is the refractive index of the fiber crosssection.

Equation (4) can be solved, as a first approximation, as an infinite waveguide, and therefore, the refractive index is supposed as a constant along the propagation axis. In this case, the electric field can be computed by the finite element method (FEM) [6]. To calculate the transversal component of the field by the FEM, it is necessary to discretize the section of the fiber in very small elements and to compute (4) for each element (see Fig. 1).

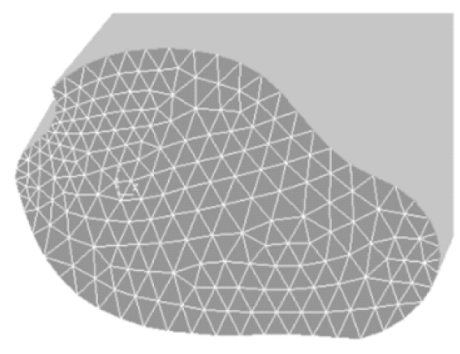

(a)

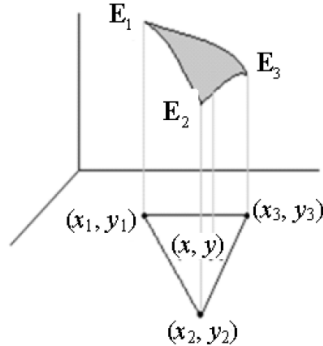

(b)
Fig. 1 Finite element method: (a) discretization in elements for the application of the method and (b) description of an element and its nodes.

After calculating the electric field $\mathbf{E}$ for each node and for all modes supported by the multimode fiber, it is necessary to superpose them in order to recover the speckle pattern. The total power can be written as [7] $P=\int_{A} I d A$, being $I=(1 / 2) c \in_{0} n|\mathbf{E}|^{2}$, the intensity of the electromagnetic wave. Thus, after summing the total power of each element of the discretization, we obtain

$$
P \approx \sum P_{e}=\frac{1}{2} c \epsilon_{0} n_{\text {core }} \sum\left|\mathbf{E}_{e}\right|^{2} A_{e}
$$

where $e$ denotes that the sum is over all elements. In our case, we simulated the behavior of a step-index 
multimode fiber Thorlabs AFS105/125Y by the implementation of the FEM in the software ComsolMultiphysics ${ }^{\circledR}$, forwavelengths $1310 \mathrm{~nm}$ and $1625 \mathrm{~nm}$ (see Fig. 2). There, it is clear that the

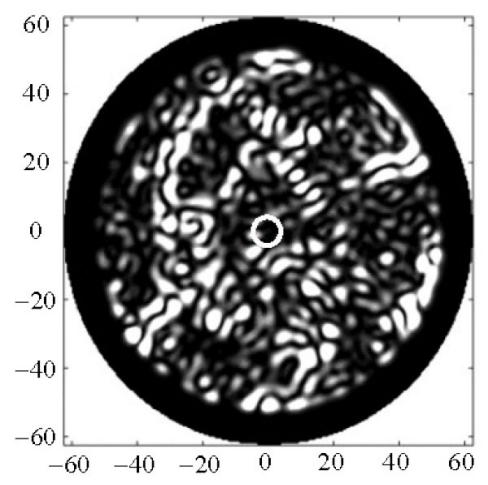

(a)

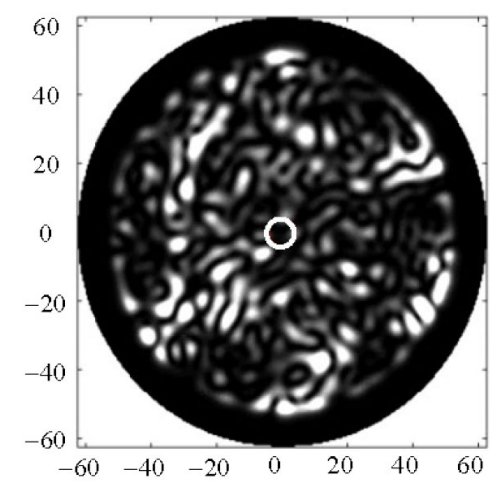

(c) number of speckle grains in a constant area (white circle) decreases when the wavelength increases, which has important effects on the dynamical behavior of this kind of sensors.

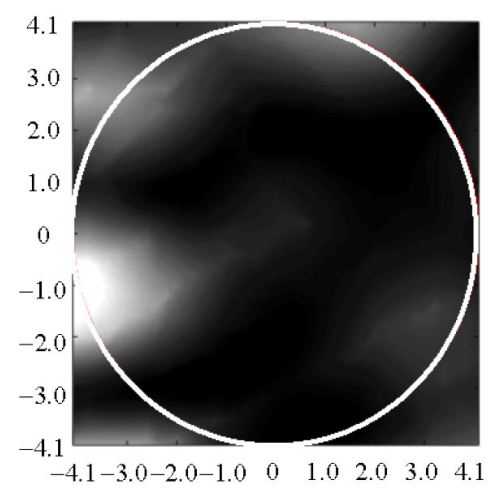

(b)

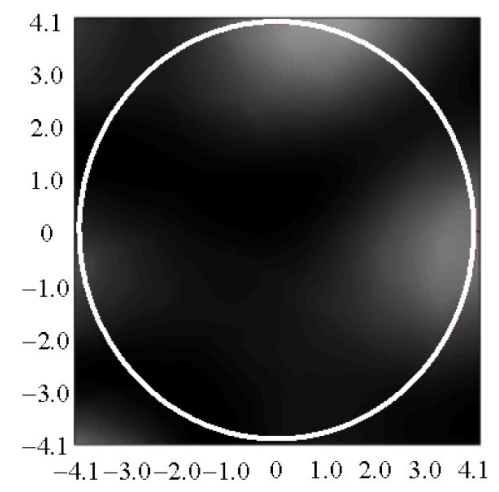

(d)

Fig. 2 Simulated speckle patterns in a step-index multi-mode fiber Thorlabs AFS105/125Y at (a) \& (b) $1310 \mathrm{~nm}$, and (c) \& (d) $1625 \mathrm{~nm}$ [the zooms of the white circles in (b) and (d) denote the detection area of a standard single-mode fiber (filter fiber) at the output of the multi-mode fiber].

\section{Experiments and results}

As mentioned in the introduction section, the non-holographic FSS was developed by arrangements of multi-single mode fibers. In our case, a 5-cm multi-mode optical fiber was located between two pieces of standard single-mode fiber, and laser sources emitting at $1310 \mathrm{~nm}, 1550 \mathrm{~nm}$, and $1625 \mathrm{~nm}$ were coupled to the sensor system (see Figs. 3,4 , and 5).

The first single-mode fiber guarantees the generation of many propagation modes in the multimode fiber which in turn generates the fiber speckle pattern. Likewise, the second single-mode fiber acts as a filter for the speckle pattern at the center of the multi-mode fiber (see Fig. 2). So, if the speckle pattern is modified due to external perturbations, the collected intensity by the second single-mode fiber will change. For a similar perturbation, changes in the collected intensity will depend on the average near field fiber speckle pattern.

When the speckle grains are small, a perturbation on the multi-mode fiber will produce a change in several speckles trapped by the single-mode fiber [see Fig. 2(d)]. If the speckle 
grains are big, the amount of grains affected by the perturbation and launched into the filter fiber are few [see Fig. 2(b)]. This effect allows modifying the reproducibility of the mechanical signal [4] and the linearity of the sensor system (see Fig. 6), only by increasing the average fiber speckle size. In this work, the average grain size was modified by changing the wavelength of the laser source.

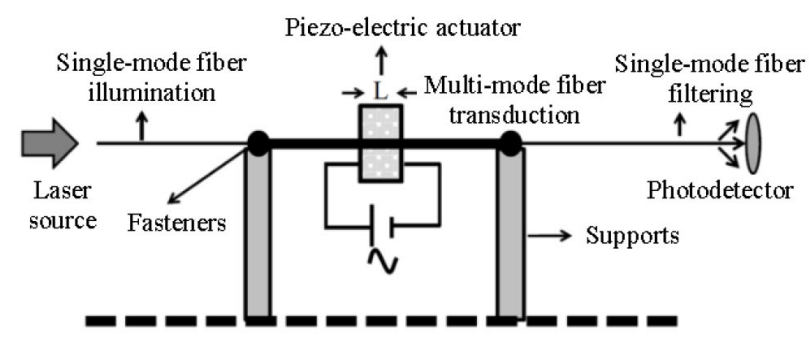

Fig. 3 Schematic drawing of the experimental setup.

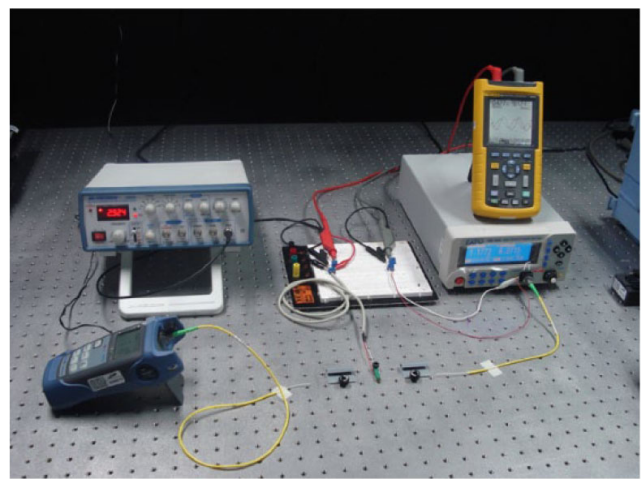

Fig. 4 Photograph of the experimental setup.

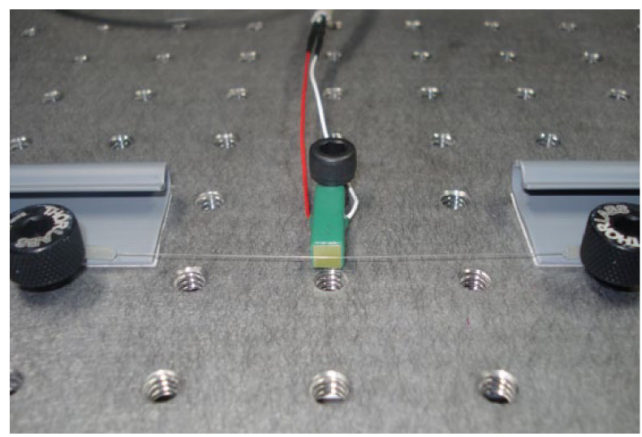

Fig. 5 Photograph of the transduction system with a multi-mode fiber submitted to a force by a piezo-electric actuator (external perturbations).

Figure 6 presents the experimental results for $1310 \mathrm{~nm}, 1550 \mathrm{~nm}$, and $1625 \mathrm{~nm}$ in the case of sine transversal dynamical perturbations of $1 \mathrm{kHz}$ being applied to the multimode fiber. These experiments were repeated twice (Test 1 and Test 2).

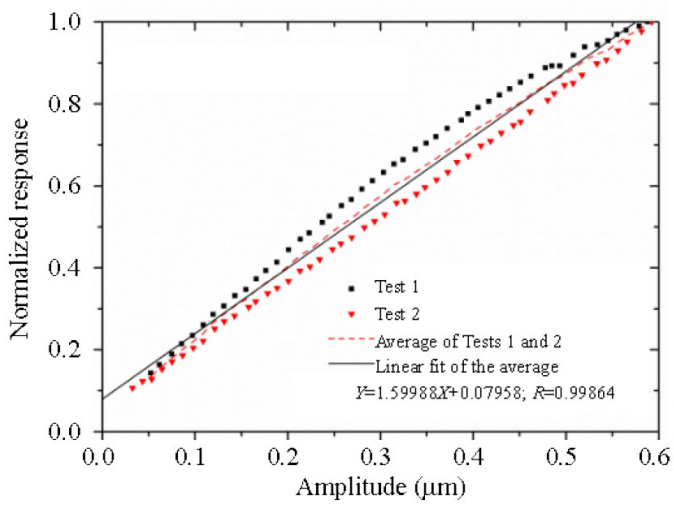

(a) Response to $1625 \mathrm{~nm}$

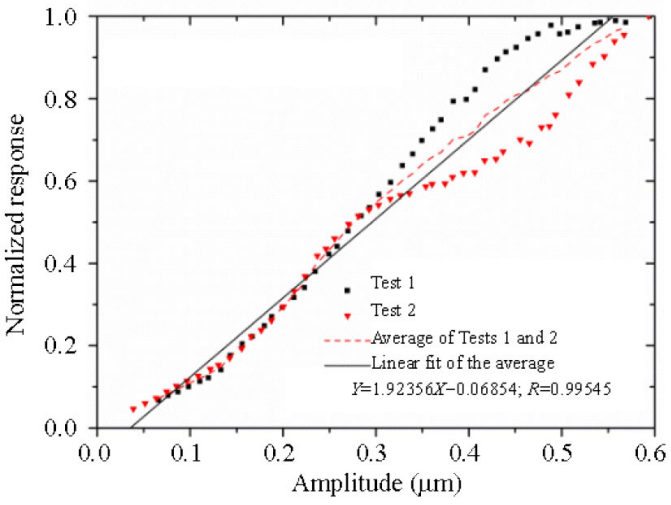

(b) Response to $1550 \mathrm{~nm}$

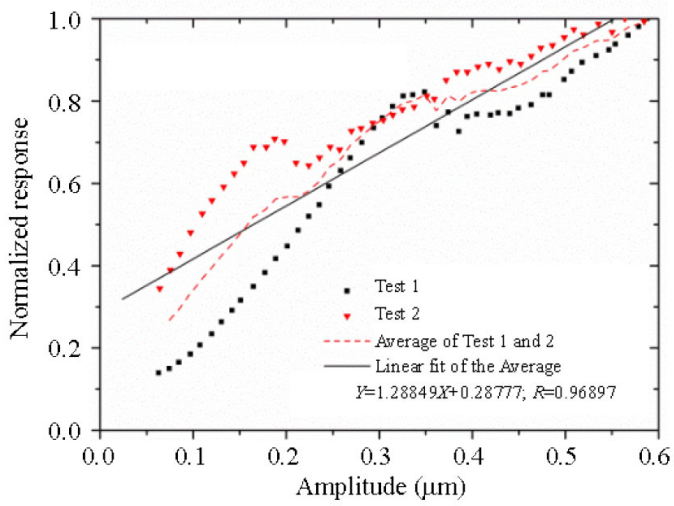

(c) Response to $1310 \mathrm{~nm}$

Fig. 6 Responses of the specklegram sensor at (a) $1625 \mathrm{~nm}$, (b) $1550 \mathrm{~nm}$, and (c) $1310 \mathrm{~nm}$.

It is evident that the linearity and the reproducibility of the sensor for higher source wavelengths were improved. This effect can be of great interest to designers and engineers.

\section{Conclusions}

In this work, preliminary results of the effects of 
varying the wavelength in a structure of a nonholographic fiber specklegram sensor were presented. In these arrangements, the speckle pattern produced by a multimode optical fiber was coupled to an optical fiber with the lower numerical aperture, which produced a filtering effect that could be used as an optical transduction mechanism. The influence of the wavelength on the sensor performance was evaluated by changing the laser source wavelength. Lasers emitting at $1310 \mathrm{~nm}, 1550 \mathrm{~nm}$, and $1625 \mathrm{~nm}$ were used, and a strong effect on the linearity of the sensor response was observed. Higher wavelengths have a correspondence with an improvement in the linearity and the reproducibility of the sensor.

\section{Acknowledgment}

A. Hoyos and J. A. Gómez acknowledge the financial support of the Politecnico Colombiano Jaime Isaza Cadavid. V. H. Aristizabal acknowledges the financial support of the Universidad Cooperativa de Colombia. Nelson Dario Gómez acknowledges the support of the Instituto Tecnológico Metropolitano (ITM). E. Rueda acknowledges the support of the Universidad de Antioquia.

Open Access This article is distributed under the terms of the Creative Commons Attribution License which permits any use, distribution, and reproduction in any medium, provided the original author(s) and source are credited.

\section{References}

[1] B. E. A. Saleh and M. C. Teich, Fundamentals of photonics. USA: John Wiley \& Sons Inc., 1991.

[2] B. Wang, C. Huang, R. Guo, and F. T. S. Yu, “A novel fiber chemical sensor using inner product multimode fiber speckle fields," in Proc. SPIE, vol. 5206, pp. 200-304, 2003.

[3] Y. Liu and L. Wei, "Low-cost high-sensitivity strain and temperature sensing using graded-index multimode fibers," Applied Optics, 2007, 46(13): 2516-2519.

[4] N. D. Gómez and J. A. Gómez, "Effects of the speckle size on non-holographic fiber specklegram sensor," Optics and Laser in Engineering, 2013, 51(11): 1291-1295.

[5] J. A. Gómez, H. Lorduy, and A. Salazar, "Influence of the volume speckle on fiber specklegram sensors based on four-wave mixing in photorefractive materials," Optics Communications, 2011, 284(4): 1008-1014.

[6] P. Torres, V. H. Aristizabal, and M. V. Andres, "Modeling of photonic crystal fibers from the scalar wave equation with a purely transverse linearly polarized vector potential," Journal of the Optical Society of America B, 2011, 28(4): 787-791.

[7] D. J. Griffiths, Introduction to electrodynamics. USA: Prentice-Hall, Inc., 1999. 